\title{
EL ARTE MODERNO EN DISPUTA. APARIENCIA Y RECONCILIACIÓN
}

\author{
María Verónica Galfione ${ }^{1}$ \\ Consejo Nacional de Investigaciones Científicas y Técnicas (CONICET) \\ Universidad Nacional del Litoral (UNL) \\ (D) https://orcid.org/0000-0003-2429-8203
}

\section{RESUMEN:}

En el presente artículos analizaremos el modo en que Dieter Henrich interpreta la concepción estética de Hegel. Como veremos, el autor considera que, durante los últimos años de su vida, Hegel revisó su posición contraria al arte moderno y desarrolló una interpretación positiva del mismo. Henrich descubre en las intuiciones de Hegel acerca del humor objetivo un punto de partida adecuado tanto para pensar una visión no soberana de la subjetividad, como para desarrollar una estética de la verdad de carácter afirmativo. No obstante, para hacerlo no solo se aparta de los supuestos de la estética hegeliana sino que, además, proyecta sobre el arte moderno y el arte contemporáneo categorías que serían propias del pensamiento tradicional. Por este motivo, tras revisar las críticas de Henrich a las teorías reflexivas de la conciencia y reconstruir el modo en que entiende la relación entre arte y subjetividad, delinearemos algunas consideraciones relativas a las dificultades que trae aparejada su tendencia a desconocer la autonomía del arte moderno en toda su radicalidad. Según sostendremos, Henrich niega los momentos críticos de la modernidad por medio de la neutralización de sus posibles excesos y a construye una teoría del arte moderno que ya no puede dar cuenta de la ambigüedad constitutiva del mismo.

PALABRAS CLAVE: Dieter Henrich; Estética hegeliana; Humor objetivo; Subjetividad estética; Modernidad.

\section{MODERN ART IN DISPUTE. APPEARANCE AND RECONCILIATION}

\begin{abstract}
ABSTRAC:
${ }^{1}$ La autora en Doctora en Filosofía, Investigadora del Consejo Nacional de Investigaciones Científicas y Técnicas y Profesora Asociada de Estética en la Universidad Nacional del Litoral (Argentina)- Email: veronicagalfione@yahoo.com.ar El arte moderno en disputa. Apariencia y reconciliación - María Verónica Galfione
\end{abstract}


In the present articles we will analyze the way in which Dieter Henrich interprets the aesthetic conception of Hegel. As we shall see, the author considers that, during the last years of his life, Hegel revised his position contrary to modern art and developed a favorable interpretation of it. Henrich discovers in Hegel's intuitions about objective humor an appropriate starting point for thinking about a non-sovereign conception of subjectivity and for developing an affirmative aesthetic of truth. To do this, however, he not only departs from the assumptions of Hegelian aesthetics but also projects traditional categories on modern art and contemporary art. For this reason, after reviewing Henrich's critiques of reflexive theories of consciousness and reconstructing the way in which he understands the relation between art and subjectivity, we will delineate some considerations concerning the difficulties that are contained in his tendency to ignore the radical autonomy of modern art. As we will argue, Henrich denies the critical moments of modernity by neutralizing his possible excesses and constructs a theory of modern art that can no longer be faithful to its constitutive ambiguity.

KEYWORDS: Dieter Henrich; Hegelian aesthetics; Objective humor; Aesthetic subjectivity; Modernity.

\section{Introducción}

Una de las líneas principales que ha trazado la filosofía alemana contemporánea remite a la posibilidad de reconstruir una concepción legítima de la modernidad por medio de la enfatización de la dimensión estética. Siguiendo las intuiciones que ya se encontraban presentes en autores como Ernst Cassirer o Alfred Bauemler (CASSIRER, 1981, 384387; BAUEMLER, 1975, 3-5), diversos pensadores han intentado reinterpretar el surgimiento del sujeto estético en términos de una ampliación o de una transformación de la noción dominante de la subjetividad. En este sentido resulta paradigmático el planteo de Dieter Henrich quien, distanciándose de la crítica heideggeriana, enfatiza el carácter antifuncionalista de la estética moderna. Para Henrich, lejos de colocar al sujeto, en tanto sub-iectum, en el centro de la reflexión y de transformar al ente en un mero estímulo sensible para la experiencia subjetiva (HEIDEGGER, 1961/ II, 195), la dimensión estética habría contribuido a desarrollar una visión no soberana de la subjetividad.

Según Henrich, una demostración de esto último puede encontrarse en las intuiciones hegelianas acerca del arte moderno. Desde la perspectiva de Henrich, durante los últimos años de su vida, ${ }^{2}$ Hegel habría

\footnotetext{
${ }^{2}$ Henrich se refiere a las Lecciones sobre estética (HEGEL, 1989) y a la reseña de los escritos póstumos de Solger (HEGEL, 1979, p. 205-235).
} 
reconsiderado la condena del arte moderno que se hallaba contenida en su famosa tesis acerca del fin del arte y habría admitido la posibilidad de un arte enfático de carácter no monumental, esto es, la posibilidad de un arte que, sin presentarse como instancia máxima de autoconocimiento del espíritu, aún mantuviese algún tipo de relación explícita con la verdad. Según sostiene Henrich, Hegel no desarrolló esta orientación de una manera acabada, pues esto no solo lo hubiese obligado a modificar la arquitectónica de las Lecciones sobre estética sino también a rechazar los supuestos metafísicos de su sistema. No obstante, la tensión que atraviesa sus reflexiones tardías acerca del arte se presenta como una crítica implícita a su propia concepción de una reflexión absoluta de la subjetividad y ofrece indicaciones importantes, entiende Henrich, acerca de cómo debería ser pensado el arte moderno y la idea misma de la subjetividad.

Por este motivo, en este artículo, tras analizar la interpretación de la estética hegeliana que propone Henrich, revisaremos la lectura no fundacionalista de la modernidad y de la subjetividad moderna que defiende este autor. Como lo pone en evidencia su propia interpretación de Hegel, para Henrich, la emergencia del fenómeno moderno de la autoconciencia sería indisociable de la revelación de la dependencia y de la finitud de ese yo que toma conciencia de sí. En este sentido, su revisión de la historia de la filosofía moderna se encontraría orientada a rechazar tanto la identificación heideggeriana del sujeto moderno con la autoafirmación del yo, como la desconfianza de la filosofía contemporánea frente a todo principio de orden subjetivo. Desde la perspectiva de Henrich, la negación del carácter omnisciente y soberano del sujeto no debería tener como consecuencia necesaria la renuncia a la capacidad unificadora del mismo. Según el autor, esta sería consustancial al desarrollo de una vida consciente y subyacería a cada una de nuestras manifestaciones subjetivas. De hecho, sería justamente este carácter irrenunciable de la subjetividad lo que reflejaría el marcado interés del pensamiento moderno por la dimensión estética. De acuerdo con Henrich, en ausencia de garantías metafísicas, ella resguardaría el poder subjetivo de integración y se presentaría como un espacio propicio para el desarrollo de interpretaciones coherentes de nosotros mismos.

Pero si, por medio de la enfatización de la dimensión estética, Henrich logra bosquejar una visión no soberana de la subjetividad, su lectura no siempre logra dar cuenta de la especificidad del arte moderno y contemporáneo. Por el contrario, su concepción del arte moderno adolece de una marcada unilateralidad, mientras que su interpretación de la producción artística contemporánea asume un carácter abstractamente descalificador. Por ello mismo, hacia el final del trabajo, delinearemos algunas consideraciones relativas a las dificultades que trae aparejado el planteo de Henrich e intentaremos evaluar los costos de su tendencia a desconocer la autonomía del arte en toda su radicalidad y a proyectar sobre el arte 
moderno y contemporáneo categorías que serían propias del pensamiento tradicional.

\section{Henrich y su defensa de la modernidad de la estética hegeliana}

Las primeras reflexiones de Henrich con respecto a la estética hegeliana datan de mediados de los años 60 y fueron presentadas originariamente en la segunda reunión del grupo "Poetik und Hemeneutik" que tuvo lugar en Múnich durante el año 1965. En esta famosa comunicación, que lleva por título "Kunst und Kunstphilosophie in der Gegenwart", Henrich procura desarrollar una interpretación modernista de la concepción estética de Hegel. A tales efectos, él identifica cuatro rasgos de la perspectiva hegeliana. En primer lugar, hace referencia al distanciamiento de Hegel con respecto a aquellas posiciones que sostenían la utopía de una epopeya de carácter moderno. Según lo entiende este autor, la insistencia de Hegel en el componente cognoscitivo del arte no lo habría llevado a admitir la posibilidad de una nueva síntesis poética que asumiera la tarea de reconciliar de una manera inmediata al sujeto con el mundo. En este punto, Hegel no solo habría aventajado a autores como Schelling, que abrigaban la expectativa de una restitución de las formas artísticas monumentales, ${ }^{3}$ sino también a aquellas perspectivas del siglo XX que todavía asumen la defensa de una estética de la verdad, esto es, por un lado, a la tradición marxista -representada particularmente por Theodor W. Adorno- y, por el otro, a la ontología existencial de Martin Heidegger. Por cierto, uno de los objetivos principales del ensayo de Henrich es demostrar que, si autores como Adorno o como Heidegger consideran que el arte solo puede conservar su verdad allí donde pone en evidencia el completo vaciamiento de la realidad o el autodominio del hombre bajo el imperio de la técnica, respectivamente, es porque continúan pensando al arte moderno sobre el trasfondo de una utopía de orden estético. Contra estos planteos, Henrich busca desarrollar, en cambio, una concepción estética que defienda el contenido de verdad de la representación artística sin obligarnos a concebir el arte en términos exclusivamente negativos, y enfatiza, por ello mismo, la insistencia de Hegel en la caducidad de las orientaciones estéticas de carácter utópico. ${ }^{4}$

\footnotetext{
${ }^{3}$ Henrich llama la atención acerca del hecho de que Hegel también se haya distanciado de las pretensiones restauradoras del pasado que tenían lugar en el ámbito propiamente artístico. Henrich tiene en mente aquí proyecto tales como el de Johann Gottfried Schadow o los de Philipe Veit y Johann Friedrich Overbeck (HENRICH, 2003, 69).

${ }^{4}$ Frente al avance de las estéticas emotivistas de origen anglosajón, el ensayo de 1965 tiene como principal objetivo reforzar aquellas posiciones que le atribuyen al arte un contenido de verdad. No obstante, Henrich considera que es necesario revisar algunos aspectos de las estéticas contemporáneas de contenido a los fines de que no sea necesario aceptar su concepción negativa del arte contemporáneo. Es decir, para Henrich, se trata de disputarle
} 
El segundo rasgo que Henrich identifica en la estética hegeliana remite a su reconocimiento del carácter reflexivo del arte moderno. Hegel habría aceptado la irreversibilidad de aquel proceso por medio del cual el arte habría dejado de ser evidente y su definición se habría transformado en un objeto de crítica y de reflexión. Desde el punto de vista de Hegel, la reflexividad no habría constituido un lastre del arte moderno, que debía ser superado por medio de la restitución de una relación inmediata con el objeto. Dicho rasgo habría sido el resultado, más bien, de la liberación del artista con respecto a toda visión preestablecida del mundo cuya génesis y cuyos límites no dependieran de la perspectiva subjetiva.

Con esta idea se encuentran asociados los dos últimos elementos de la estética hegeliana que son recalcados por Henrich; esto es, la afirmación de la libre disponibilidad de todas las formas artísticas del pasado, por un lado, y la caracterización del arte moderno en términos de un arte de naturaleza parcial, por el otro. La primera idea remite al rol determinante que asume la subjetividad del artista moderno en la elección de los temas y de los estilos de configuración. El artista "deviene creador de sus contenidos en la medida en que estos no le son fijados como tareas vinculante" (HENRICH, 2003, 71) y este hecho se ve reflejado, dice Henrich, en la distancia reflexiva que se establece entre el sujeto creador y la obra efectivamente producida.

El concepto de parcialidad, en cambio, es asociado por Henrich a las consideraciones de Hegel con respecto a la tendencia histórica hacia una superación de la representación artística como medio adecuado para la manifestación de la verdad. De acuerdo con Henrich, la concepción hegeliana de la parcialidad del arte moderno no solo se encontraría ligada a la cualidad sensible de la representación artística. De ser así, sería posible pensar que el arte moderno puede continuar representando en la intuición sensible los mismos contenidos que el espíritu ha llegado a aprehender en términos conceptuales. Según Henrich, lo que resultaría determinante sería, más bien, el grado de abstracción que asume el mundo moderno en virtud de la primacía de la subjetividad. Sería justamente en virtud de esta abstracción que el arte se vuelve incapaz de representar el conjunto de la realidad "como un plexo de carácter racional" (HENRICH, 2003, 133) y se ve obligado a

la estética de contenido tanto a la tradición marxista como a la escuela hermenéutica que nace con el pensamiento de Heidegger (HENRICH, 2003, 128s).

${ }^{5}$ En "Zerfall und Zukunft. Hegels Diagnose über das Ende der Kunst", un ensayo del 2002, en el cual Henrich reelabora un trabajo de 1996, el autor cuestiona la posibilidad de remitir esta abstracción a procesos de orden exclusivamente material. Esta mala interpretación de la tesis hegeliana respondería a la interferencia de la perspectiva marxista. La "correcta" interpretación de dicha tesis es expuesta por Henrich en los siguientes términos: "La idea se realiza en la modernidad por medio de procesos cuyo rasgo esencial remite al hecho de que tienen lugar a través de transformaciones en la relación con uno mismo y en las actitudes de los sujetos. La presentación de estas transformaciones, junto con su fundamento, no puede 
tratar con recortes parciales de la realidad. Por este motivo, sostiene Henrich, "el arte ya no habría sido para Hegel un modo de presentación de la verdad para la conciencia". Desde su punto de vista, en las Lecciones sobre estética el arte moderno se presentaría "como una consecuencia de la verdad"; él "presupondría el saber reflexivo de la unificación realizada y completa de la conciencia y de la realidad y se movería en el marco del mismo... No obstante, el arte sería incapaz de reproducir los fundamentos y el contenido de la completa mediación de conciencia y realidad" (HENRICH, 2003, 133).

Para Henrich, esta interpretación de la representación artística en términos de una consecuencia de la verdad hubiese permitido que Hegel revisara la arquitectónica de sus lecciones sobre estética y que incorporara en ellas sus intuiciones tardías acerca del arte moderno. Henrich tiene en mente aquí las referencias que hace Hegel durante el último ciclo de sus lecciones sobre estética en Berlín (1828/29) a ciertas obras emblemáticas del mundo moderno. Entre ellas se encuentran los poemas de Friedrich Rückert y, sobre todo, El Diván de Occidente, de Goethe. ${ }^{6}$ No obstante, esta interpretación hubiese traído aparejada, además, la renuncia a la dignidad ontológica de la representación artística, la cual, como es sabido, se hallaba asociada según Hegel a la definición del arte como manifestación sensible de la verdad. Es decir, entender al arte como una consecuencia de la verdad hubiera permitido que Hegel evadiera la condena del arte del presente, que aparentemente se sigue de sus lecciones sobre estética, pero lo hubiese obligado a renunciar a su carácter enfático. El arte moderno se hubiese presentado como un arte eminentemente lúdico, sin relación explícita con la verdad.

ser llevada al plano de la intuición, esto es, a aquel plano que sería constitutivo de la obra de arte." (HENRICH, 2003, 70)

6 Desde su perspectiva, Hegel habría sido positivamente influenciado por diversas manifestaciones artísticas de su época, y se habría visto movido a desarrollar, por ello mismo, una concepción estética que diera cuenta de ellas de una manera auténtica. No obstante, por falta de tiempo, estas ideas habrían quedado inconclusas en el momento de su muerte. La tesis del fin del arte sería central hasta 1828. Annemarie Gethmann-Siefert discute el intento de Henrich por desplazar la tesis acerca del fin del arte. A tales efectos, la autora se basa en el análisis pormenorizado de las diversas versiones de las lecciones de estética. Desde su punto de vista, ya Hotho habría intentado negar la importancia de la tesis acerca del fin del arte y lo habría hecho a los fines de fundamentar la posibilidad de un renacimiento del arte clásico de corte germánico. Pero si Gethmann-Siefert no está de acuerdo con Henrich en este punto, la autora considera, al igual que él, que la inclinación de Hegel por cierto tipo de obras de arte moderno se habría fundado en argumentos de orden extraestético. El arte moderno que Hegel reivindicaría sería aquel que se aparta del ideal de la autonomía del arte: "un arte dedicado al goce, la posibilidad de un arte que, por medio de la belleza, haga sensible a los hombres para el significado cultural de su contenido, de sus proclamaciones, y que la posibilidad de una mediación poética de lo extraño en lo propio por medio del arte" (GETHMANN-SIEFERT, 2005b: 45). Volveremos a este punto para el caso de Henrich. 
El intento de Henrich por salvar los elementos modernistas de la teoría de Hegel, dejando de lado sus aspectos perimidos, se encuentra orientado a superar la propia tensión a la que conducirían las consideraciones hegelianas acerca del arte moderno y a hacer posible una teoría estética que evada los dos grandes peligros de la estética contemporánea; esto es, una estética que, por una parte, no obligue a asumir una definición del arte en términos de verdad a costa de renunciar al arte moderno -como plantearían autores como Hans Seldmayr- o de definirlo en términos negativos - como sostiene Adorno- ${ }^{7}$, y que, por la otra, no lleve a defender el arte moderno sobre la base de un corrimiento más allá del plano de la verdad -como querrían las teorías contemporáneas de corte emotivista. Por ello mismo, la interpretación de la estética hegeliana que desarrolla Henrich no solo conlleva una fuerte revisión de la arquitectónica de las lecciones sobre estética y trae aparejado un desplazamiento de la centralidad de la tesis acerca de la muerte del arte moderno. Además de torcer la rigidez del sistema hegeliano a los fines de permitir el ingreso del arte moderno, Henrich debe hacer posible el reconocimiento de su valor cognoscitivo. Desde la perspectiva de Henrich, esto último puede lograrse si se abandonan los supuestos que sostienen el proyecto hegeliano de una refundación subjetiva de la unidad de la realidad con la razón. De manera tal que, si Henrich puede atribuirle un carácter enfático a un tipo de arte que ya no puede ser interpretado en términos de manifestación sensible de la idea, es decir, al arte moderno, es porque primero rechaza la confianza de Hegel en la posibilidad de pensar la relación absoluta consigo mismo como un proceso mediador de la sustancia y el sujeto. ${ }^{8}$

\footnotetext{
${ }^{7}$ La referencia a la estética de Hegel le sirve a Henrich, en primer lugar, para demostrar que es posible defender una estética moderna de la representación sin verse obligado a entender el vínculo con el presente en términos de deformación y decadencia. En segundo lugar, el autor busca desactivar por medio de su relectura de Hegel aquellas perspectivas que, a los fines de asumir una comprensión sustancialista de la realidad, le atribuyen al arte moderno un carácter inesencial. Si en el primer caso, Henrich hace referencia a Adorno, en el segundo se refiere a la postura de Hans Sedlmayr. Para este autor, el arte moderno compartiría la culpa de la modernidad de haber disuelto en el mundo material la analogia entis del espíritu y de haber renunciado, de esta forma, a la unidad de la materia y la forma (HENRICH, 2003, 144). Frente a estas dos posturas, Henrich sostiene "La representación puede ser de carácter parcial y resultar compatible con la apertura, la reflexividad y la formación histórica del artista moderno. La realidad que el arte hace visible debe poder ser interpretada no como realidad en un sentido universal" (HENRICH, 2003, 134).

${ }^{8}$ Existen intérpretes que consideran que Henrich se equivoca en este punto. Así, Slavoj Žižek sostiene la diferencia crucial entre Hegel y Henrich se encuentra en otra parte. Desde su punto de vista, también Hegel admitiría la existencia de un excedente, pero lo ubicaría en el extremo opuesto a Henrich, "no en un "autoconocimiento" interior, previo a la distancia reflexiva, autoobjetivante del sujeto respecto de sí mismo, sino en una externalidad radicalmente contingente de algún residuo material, inerte y no racional. Este objeto es el correlato del sujeto: confiere a este último el mínimo de consistencia y con ello impide que caiga en el abismo del círculo vicioso.” (ŽIŽEK, 1994, 111s.)
} 


\section{Aspectos esotéricos de la modernidad de la estética de hegeliana}

Como ya vimos, el componente propiamente moderno que se hallaría contenido en la estética hegeliana remitiría, según Henrich, a la renuncia a la expectativa de una nueva síntesis artística que fuera capaz de restablecer la unidad inmediata entre el individuo y el mundo. No obstante, la reticencia de Henrich con respecto a la posibilidad de pensar el arte moderno en términos utópicos resulta dependiente de una concepción claramente antihegeliana de la subjetividad y de la verdad. A diferencia de Hegel, Henrich no considera que la verdad haya dejado de manifestarse de una manera adecuada en el arte moderno porque el sujeto ha llegado a aprehenderla en términos conceptuales. Para él, la parcialidad del arte moderno, lejos de encontrarse ligada al logro filosófico de una reflexión absoluta de la subjetividad, esto es, lejos de hallarse asociada al carácter pasado del arte, se deriva, más bien, del descubrimiento de la dependencia de la subjetividad con respecto a un fundamento que no se halla bajo su poder ni resulta cognoscible en términos reflexivos. De manera tal que, si el absoluto ya no se manifiesta plenamente en el arte moderno no es porque la intuición sensible haya sido superada en el plano de la filosofía, sino porque la subjetividad ha advertido, más bien, hasta qué punto ella misma hunde sus raíces en un fundamento que no puede ser agotado por medio de la reflexión.

Aquí tocamos un aspecto central de la filosofía de Henrich, que recorre la totalidad de su obra, desde su reseña crítica de la segunda edición de Kant y el problema de la metafísica, de Heidegger (HENRICH, 1955, 2869), hasta sus discusiones más actuales con la filosofía analítica de la mente (HENRICH, 1982, 93; 2007, pp. 1-19). ${ }^{9}$ Nos referimos concretamente al problema del doble carácter de la subjetividad en tanto instancia que se encuentra remitida a sí misma y que es incapaz de pensar su fundamento en términos externos, por una parte, pero que se halla imposibilitada, por otra parte, de acceder a dicho fundamento por medio de la reflexión. A este doble carácter de la subjetividad, Henrich se refiere en los siguientes términos:

\footnotetext{
En este sentido solo existimos en la medida en que poseemos ese saber de nosotros mismos. No obstante, ese centro de nuestra existencia, que presuponemos cada vez que comprendemos algo o que actuamos, no se nos presenta él mismo como algo claro y evidente. Él es ciertamente el
}

\footnotetext{
9 Frank realiza un buen resumen de los debates que mantuvo lo llamada escuela de Heidelberg (FRANK, 2013, 1- 21). Cabe aclarar en este punto que, además de Henrich, la escuela de Heidelberg incluiría a autores como Ulrich Pothast, Konrad Cramer, Jürgen Stolzenberg, Manfred Frank o Stefan Lang.
} 
presupuesto, pero también el límite de toda nuestra comprensión. (HENRICH, 2003, 16) ${ }^{10}$

En el texto sobre Hegel de 1965 este problema es enunciado por Henrich por medio de la referencia a los conceptos de "autoconciencia" y "autoconservación". Al igual que en "Fichtes ursprüngliche Einsicht" (1967), la utilización que hace aquí Henrich de estos conceptos se encuentra orientada a discutir el diagnóstico que realiza Heidegger acerca del carácter monolítico y decadente de la filosofía moderna. Según Henrich, la identificación heideggeriana de la conciencia moderna con la progresiva radicalización del poder propio de la subjetividad y de su dominio sobre el ente en cuanto tal, perdería de vista el hecho de que, si el sujeto debe conservar su propia existencia, es porque el fundamento de esta última no se encuentra bajo su poder. ${ }^{11}$ En este sentido, la emergencia de la conciencia de sí resulta indisociable del descubrimiento del carácter dependiente de aquel sujeto que encuentra en sí mismo su fuente última de determinación. El sujeto toma conciencia de sí en la medida en que se apercibe de la necesidad de garantizar su propia subsistencia, esto es, en la medida en que advierte que, aun cuando su existencia no dependa de una instancia de carácter externo, tampoco se encuentra plenamente en su poder. Por eso mismo, el relato de la filosofía moderna que, partiendo de la recuperación de la noción estoica de autoconservación que tiene lugar en el siglo XVII y llegando hasta los orígenes del idealismo alemán, construye Henrich, pone en evidencia que "ya en la antropológica temprana, la experiencia de solo poder conservarse e incrementarse en función de uno mismo, se encuentra vinculada con la conciencia de no disponer de la propia existencia." (HENRICH, 2003, 136)

Desde la perspectiva de Henrich, la doble naturaleza del sujeto reflexivo da lugar a una situación paradójica. En efecto, la relación con uno mismo, que presupone el imperativo de la autoconservación, hace emerger la pregunta acerca del fundamento del yo. No obstante, este fundamento ya no puede ser pensado como algo externo que produce al sí mismo en vistas a una relación teleológica más elevada. Al respecto, sostiene Henrich,

Toda posible ontología debe ser probada por medio de la reflexión de sí y esto deja en claro que el concepto de finalidad solo puede ser definido a partir de la relación reflexiva de la vida. De esta forma, la experiencia según la cual el sí mismo, en función de su propio principio interno, solo se encuentra vinculado con él mismo, no solo tiene como complemento la

\footnotetext{
${ }^{10}$ En otro contexto Henrich hace referencia a la distancia que media entre "la certeza acerca de uno mismo y la incertidumbre acerca de lo que somos en realidad" (HENRICH, 2016, 21).

${ }^{11}$ Henrich se dedica en profundidad a este tema en „Die Grundstruktur der modernen Philosophie“, un ensayo de 1966 que sería publicado en 1976 acompañado de un texto complementario (HENRICH, 1976, 97 - 144).
} 
certeza de que su existencia depende de un fundamento insondable sino también el saber con respecto al carácter necesariamente inescrutable de ese fundamento para la conciencia. (HENRICH, 2003, 136)

En este sentido, la doble naturaleza del sujeto reflexivo conduciría de manera inexorable a una situación que, siguiendo la expresión de Manfred Frank, podríamos caracterizar en términos de un "estado de necesidad cognoscitiva" (FRANK, 1989, 33). Es este estado de necesidad cognoscitiva, que sería inherente al sujeto moderno en tanto sujeto reflexivo, el que justifica, para Henrich, la importancia tanto del pensamiento metafísico ${ }^{12}$ como de la representación artística. En ambos casos, de lo que se trataría sería de integrar el carácter contradictorio de la autorreferencialidad del sí mismo, ${ }^{13}$ por un lado, con la experiencia de la dependencia del yo con respecto a un fundamento que no se encuentra a su disposición y que resulta inaccesible para la conciencia, por el otro.

De acuerdo con Henrich, esta manera propiamente moderna de entender el arte se ve reflejada en las intuiciones de Hegel acerca del humor objetivo, como forma característica del arte romántico. De hecho, Hegel se habría valido de esta expresión para hacer referencia a la capacidad de algunas obras literarias de su época para ofrecernos una representación indirecta y no objetivadora de un contenido profundo. En este sentido, el humor objetivo no sería menos dependiente que el humor subjetivo -o ironía- de la elevación de la subjetividad por sobre toda posible realidad. Ambos se encontrarían hacia el final de la forma romántica del arte y se definirían, por ende, en función del primado de la subjetividad. No obstante, si la ironía se caracteriza por negar los aspectos sustanciales de la vida de

\footnotetext{
${ }^{12}$ La defensa que hace Henrich de la metafísica ha dado lugar a una importante disputa con Habermas. Esta disputa puede retrotraerse hasta 1974, año en el cual ambos filósofos se encontraron a raíz de la entrega a Habermas del Hegel-Preis de la ciudad de Stuttgart. En dicha ocasión, Habermas se dirigió implícitamente contra Henrich al sostener el carácter incompatible de la metafísica con las sociedades modernas. (HABERMAS/HENRICH, 1974, 48). La posición de Henrich ante este problema aparecerá en Fluchtlinien (HENRICH, 1982), un libro que fue escrito en 1981 y que fue duramente reseñado por Habermas en 1985 en la revista Merkur (HABERMAS, 1985, 898-905). Esta reseña será publicada nuevamente en Nachmetaphysisches Denken (HABERMAS, 1988) bajo el título "Rückkehr zur Metaphysik? Eine Sammelrezension". En este libro también aparecerá la respuesta de Habermas a "Was ist Metaphysik? - was Moderne? Zwölf Thesen gegen Jürgen Habermas", un texto en el cual Henrich formulaba con detenimiento su concepción de una metafísica de carácter moderno (HENRICH, 1988, 11-43). La respuesta de Habermas llevará por título "Metaphysik nach Kant" y también será incluida en Nachmetaphysisches Denken.

${ }^{13}$ Para un desarrollo más acabado del concepto de integración es posible consultar el ensayo de Henrich "Selbstbewusstsein und spekulatives Denken" (HENRICH, 1982). La tarea de la integración consistiría en "reunir entre sí las perspectivas fundamentadas y las preguntas irresueltas, pero inevitables, de tal manera que se dé lugar a un todo que ya no pueda traer aparejadas nuevas preguntas radicales". (HENRICH, 1982, 125)
} 
los hombres, el humor objetivo solo se vuelve contra la imagen desfigurada de estos últimos. Por ello mismo Hegel identificaría el humor subjetivo con el mero juego de las ocurrencias subjetivas, mientras que, en el caso del humor objetivo, el juego de la fantasía resultaría dependiente de un criterio de orden espiritual. Al respecto sostiene Henrich: "El humor lleva a cabo su relación negadora de lo real-finito solo en la medida en que lo finito puede ser considerado finalmente como algo en sí mismo nulo, que no tiene existencia como una forma de manifestación de realidad del espíritu." $(\text { HENRICH, 2003, 85) })^{14}$

Como puede apreciarse, el eje de la literatura humorística no se encontraría, según Hegel, en los contenidos representados sino en el tratamiento literario de los mismos. Por este motivo, sostiene Henrich, el efecto humorístico dependería, para Hegel, de "los vaivenes del humor". Estos no solo generarían una sensación agradable en la subjetividad del lector, que también es propenso a dichos movimientos, sino que, "en su insignificancia" podrían cumplir, además, una auténtica tarea formativa. Desde la perspectiva de Hegel, esto sucedería de una manera paradigmática en las comedias de Aristófanes y de Shakespeare y en los Lebensläufe in aufsteigender Linie de Hippel, en la medida en que, en tales obras, el autor no se abandonaría al azar de sus ocurrencias y dotaría a la obra "de espíritu y de profundo sentimiento" (HEGEL, 1989, 215). En este punto, Henrich hace referencia a la afirmación de Hegel, según la cual el "vagar totalmente ingenuo, ligero, inaparente" de un autores como Sterne o como Hippel, ofrecería "precisamente el supremo concepto de profundidad; y puesto que son precisamente singularidades que afloran desordenadamente, tanto más profundamente debe la conexión interna estar asentada y resaltar en lo singularizado como tal el punto luminoso del espíritu." (HEGEL, 1989, 441; HENRICH, 2003, 86) Henrich reconoce que Hegel no aclara mayormente a qué haría referencia esta "profundidad", este "punto luminoso del espíritu". No obstante, asevera que este contendido seguramente se encontraría vinculado con aquella reconciliación de la subjetividad consigo misma que tendría lugar en las comedias de Aristófanes. Más aún, para Henrich, en coincidencia con su propia concepción de la subjetividad, Hegel asumiría aquí la imposibilidad de una representación directa de la subjetividad y le atribuiría al arte la tarea de resguardar la experiencia prerreflexiva de la identidad. Al hacer referencia al "punto luminoso del espíritu" Hegel estaría suponiendo, dice Henrich, que "en la profundidad existe una relación para estas singularidades, la cual solo puede ser intuida por el humorista y presentada de una manera meramente indirecta" (HENRICH, 2003, 86). El arte se presentaría así como un sucedáneo de la reconciliación. Bajo la forma del humor objetivo, él nos brindaría la certeza de que, en última

\footnotetext{
${ }^{14}$ Sin mencionar el desplazamiento, Henrich utiliza aquí la descripción que hace Hegel de la comedia griega (HEGEL, 1989, 880-883; GETHMANN-SIEFERT, 2005a, 175-188).
} 
instancia y aun me medio de lo ocasional, el mundo no se caracteriza finalmente por la escisión.

No obstante, justo aquí resulta particularmente visible la distancia que separa el planteo de Henrich de la perspectiva de Hegel. De hecho, allí donde este confiaba en la posibilidad de una mediación total entre el sí mismo y el ser, Henrich apuesta a desarrollar un tipo de reflexión filosófica que asuma el carácter insondable de dicha mediación. Por ello mismo, el arte no sería para Henrich la manifestación sensible del absoluto, pero tampoco poseería un carácter meramente lúdico, en tanto forma superada por la marcha del espíritu por el mundo. El arte mantendría, más bien, una relación de paridad con la filosofía en la medida en que ninguna de las dos instancias lograría eliminar la diferencia que existe entre lo mediado y el proceso de mediación.

A continuación revisaremos, en primer lugar, la crítica que dirige Henrich a aquellas perspectivas filosóficas que intentan dar cuenta en términos reflexivos del fundamento de la conciencia. Esto nos obligará a hacer una breve alusión al concepto de familiaridad prerreflexiva con uno mismo, por medio del cual Henrich procura integrar la referencia a sí mismo, que sería propia de la autoconciencia, con la experiencia, que trae aparejada la necesidad de la autoconservación, de la dependencia de un fundamento que no se encuentra a disposición de la conciencia ni resulta comprensible por ella. En segundo lugar, analizaremos el modo en que Henrich concibe el rol específico que asume la dimensión artística en el marco de la configuración de una vida de carácter consciente. En este contexto, nos referiremos especialmente a la manera en la que este autor explica la distancia que media entre el arte clásico y el arte moderno.

\section{Henrich y la subjetividad}

Ya desde mediados de los años 60, uno de los objetivos principales de la reflexión filosófica de Henrich es discutir aquellas perspectivas que, en su afán de garantizar la autofundamentación de la subjetividad, explican el autoconocimiento primero del yo en términos de un giro reflexivo sobre sí mismo. Desde el punto de vista de Henrich, esta manera de entender la relación del yo consigo mismo se encuentra atravesada por una serie de contradicciones que socavan desde el comienzo la posibilidad de que el yo se convierta en el principio último del conocimiento del mundo. Para Henrich, fue Fichte el primer filósofo moderno que llegó a advertir la magnitud de las dificultades que se derivaban de la interpretación reflexiva de la autoconciencia, y que intentó desarrollar una concepción alternativa de la misma. En este punto, habría resultado determinante el hecho de que Fichte hubiese estado más interesado en indagar la compleja estructura de la autoconciencia que en establecer un fundamento certero para la filosofía. De hecho, por ese motivo, a diferencia de pensadores tales como Descartes o 
como Leibniz, (HENRICH, 1966, 190s), ${ }^{15}$ Fichte habría sido capaz de percibir el carácter inconsistente de aquellas perspectivas que procuraban explicar el fenómeno de la autoconciencia en términos de una vuelta reflexiva del yo sobre sí mismo. ${ }^{16}$ Para Fichte, estas perspectivas o bien procedían de una manera circular, o bien caían en un regreso al infinito. En el primer caso, la argumentación de Fichte buscaba poner en evidencia que, para explicar en términos reflexivos el autoconocimiento del yo, era necesario suponer que este último ya contaba con un conocimiento de sí que antecedía a la propia instancia de la reflexión. Esto es, sin este supuesto no era posible dar cuenta de la capacidad del yo para identificarse con aquella representación de sí que obtenía por medio de la reflexión. (CRAMER, 1970, 563; FRANK, 1983, 251)

El segundo argumento crítico fue formulado por Fichte en Versuch einer neuen Darstellung der Wissenschaftslehre, una obra que, desde la perspectiva de Henrich, se encontraba explícitamente orientada a superar la deficiencias que ofrecía la primera formulación del principio fundamental de la Wissenschaftslehre. En dicha obra, Fichte evaluaba la tesis kantiana según la cual toda representación de algo tenía como presupuesto un representarse a sí mismo y sostenía que, si interpretábamos esta tesis de tal manera que cada estado de conciencia debía volverse objeto de un nuevo estado de conciencia, entonces necesariamente nos veíamos embarcados en

\footnotetext{
15 Más adelante, Henrich revisa esta postura y rechaza aquellas interpretaciones que sostiene que Descartes concibe la subjetividad en términos de autotransparencia y autoinstitución. Según sostiene Henrich en Denken und Selbstsein: „El punto de partida del asegurarse cartesiano de sí es la referencia a uno mismo en el pensamiento y ciertamente en el peculiar estado de la duda... De esta forma, la certeza es vinculada a la aparición de la duda. La certeza de uno mismo queda relacionada así con el saber de la existencia de límites en la esencia misma de aquel ser que se encuentra en tal estado de autocerteza." (HENRICH, 2007, 25)

${ }^{16}$ Estas críticas a la teoría reflexiva de la conciencia son compartidas con las corrientes autorrepresentacionalistas (self-representationalism) de la actual filosofía de la mente. Dichas corrientes se encuentran representadas por autores tales como Terence Horgan, Tomis Kapitan, Uriah Kriegel y, con algunas variaciones, también por Kenneth Williford y Dan Zahavi. Estos autores definen los actos o vivencias conscientes como aquellos que, junto a su objeto, se representan a sí mismos. No obstante, ellos rechazan la posibilidad de que este representarse a sí mismo sea entendido como una representación en un nivel superior y lo ubican, más bien, en un mismo plano. Esto es, al igual que Henrich, el autorrepresentacionalismo rechaza la teoría reflexiva de la autoconciencia (aquí denominada teoría de los dos niveles), en la medida en que considera que ella da lugar a desarrollos circulares y regresivos. Sin embargo, el autorrepresentacionalismo entiende que la representación es un concepto básico de una teoría del espíritu y se aparta en este punto de las reflexiones de Henrich. Para este, y para la escuela de Heidelberg en general, la representación es una relación de dos partes y da lugar, por ello mismo, a una serie de contradicciones. En cualquier caso, en el marco de la propia filosofía de la mente se pueden encontrar posiciones más cercanas a la de Henrich en autores como Hector Neri Catañeda y Sydney Shoemaker. (FRANK, 2013, 1- 21).
} 
una reflexión de carácter infinito. Fichte describía esta dificultad de la siguiente manera:

para volverte consciente de tu pensar, debes ser consciente de ti mismo. Eres consciente de ti mismo, dices. Diferencias entonces necesariamente tu yo pensante del yo pensado en el pensamiento. Pero para poder hacer esto, el yo pensante en aquel pensamiento debe volverse objeto de un pensamiento más elevado, para poder volverse objeto de la conciencia; y así obtienes un nuevo sujeto, que se vuelve consciente de aquel que hasta el momento había sido la autoconciencia. Aquí argumento de nuevo como antes y sostengo que, una vez que hemos comenzado a proceder de acuerdo con esta ley, ya no es posible que encontraremos nunca más un lugar en el cual podamos detenernos. Para cada conciencia necesitaremos hasta el infinito una nueva conciencia cuyo objeto sea la primera y así nunca llegaremos a poder asumir una verdadera conciencia. (FICHTE, 2014, 106)

No obstante, como ya sabemos, nada podría encontrarse más alejado de la posición de Henrich que el intento de abandonar, en función de estas conclusiones, la referencia al principio de la subjetividad. Para Henrich, el carácter insoluble de las contradicciones a las que conducen las concepciones reflexivas de la autoconciencia pone en evidencia el desacierto de las perspectivas fundacionalistas de la filosofía moderna, esto es, de aquellas perspectivas que, dejando de lado la experiencia de la necesidad de autoconservación, intentaban garantizar la autofundamentación de la subjetividad (DEWS, 1995: 169s). En este contexto, Henrich advierte que las dificultades que se seguían de las teorías reflexivas modernas de la autoconciencia -y que persistirían en ciertas concepciones contemporáneas de la filosofía de la mente ${ }^{17}$ - se encuentran vinculadas a su tendencia a atribuirle un carácter relacional al autoconocimiento del yo y a concebirlo, por ende, como un caso particular de la conciencia de orden objetual. Por ello mismo, a la hora de buscar una alternativa que permita integrar la autorreferencialidad de la subjetividad con la experiencia de su falta de soberanía, Henrich recurre a la figura de una familiaridad prerreflexiva y preproposicional con uno mismo. ${ }^{18}$ Desde su perspectiva, esta figura

\footnotetext{
${ }^{17}$ Ver nota anterior.

18 A este tenerse a sí mismo prerreflexivo Henrich lo llama „Vertrautheit mit sich selbst” (Cf. HENRICH, 1970, 257-284). Frank adoptará también esta expresión ( $C f$. FRANK, 1993, 448-9; 1995, 156).
} 
evadiría el momento relacional, que presupondrían las teorías reflexivas y en función del cual se habría pretendido objetivar aquello que se encuentra más allá del poder y de la capacidad comprensiva de la subjetividad. Por lo demás, de esta forma sería posible superar la falsa disyuntiva que plantea la filosofía contemporánea y que obliga a elegir entre la admisión de una subjetividad transparente e instituyente y la disolución de la subjetividad en un conjunto de poderes anónimos e irracionales.

Sin embargo, no habría sido Fichte el encargado de sacar las conclusiones de esta intuición relativa al carácter prerreflexivo de esta familiaridad primitiva con nosotros mismos. Según Henrich, al intentar explicar la autoconciencia por medio de la teoría filosófica, Fichte habría vuelto a enredarla en el proceso de la reflexión. Por ello mismo, Henrich recurre al pensamiento de Friedrich Hölderlin, en la medida en que este le ofrece una concepción filosófica que es capaz de dar cuenta de una manera no teórica de la carencia que se encontraría en la base de la existencia subjetiva. En este punto el fundador de la escuela de Heidelberg apela a "Urtheil und Seyn", el famoso fragmento de 1795 en el cual el joven poeta ponía de relieve la dependencia de todo juicio con respecto al presupuesto de un ser de carácter indivisible. Dicho brevemente, lo que Hölderlin hacía en dicho fragmento era analizar la composición de la palabra Ur-Theil a los fines de interpretar el juicio (Urtheil) en términos de una partición originaria (Ur-Teilung) y de postular, en tal sentido, la existencia de una unidad que se encontraría más allá de la separación que se establece en el marco del pensamiento conceptual. ${ }^{19}$ "Juicio es en el más alto y estricto sentido la originaria separación del objeto y el sujeto unidos del modo más íntimo en la intuición intelectual, es aquella separación mediante la cual -y sólo mediante ella- se hacen posibles objeto y sujeto, es la partición originaria." (HÖLDERLIN, 1983, 26) ${ }^{20}$

En este lugar no podemos indagar de una manera acabada hasta qué punto la posición de Henrich presupone la existencia de una instancia de carácter absoluto que operaría de base de nuestra relación consciente con nosotros mismos. No obstante, resulta necesario hacer una breve mención a las dificultades que conllevaría la defensa de una lectura semejante. De hecho, pese a las resonancias premodernas de la perspectiva de Henrich, su propia insistencia en la autorreferencialidad de la instancia subjetiva obliga a descartar la pretensión de interpretar su planteo en términos ontológicos. Al respecto, conviene recordar la propia afirmación de Henrich, según la cual el principal problema de la filosofía moderna habría sido "pensar la relación con uno mismo de la vida subjetiva de tal manera que la

\footnotetext{
${ }^{19} U r$ : origen, teilen: partir, dividir.

${ }^{20}$ Henrich analiza en diversos artículos el modo en que, tras un período de fuerte cercanía durante la estancia de ambos en la ciudad de Frankfurt, Hegel se aparta de la concepción de Hölderlin acerca del carácter parcial del proceso reflexivo de la conciencia. (HENRICH, 1990, 11-64)
} 
representación de su fundamento inextricable no se presentase como una segunda instancia junto a ella, sino en el marco de la relación con uno mismo y como momento de la propia esencia." (HENRICH, 2003, 137)

En este sentido, no sería posible pensar que Henrich se encuentre dispuesto a admitir la existencia de un principio ontológico que opere de fundamento de nuestra vida consciente. Si el autor enfatiza el carácter insondable del fundamento de nuestra vida consciente, el momento oscuro que sería inherente a nuestra relación con nosotros mismos, no lo haría a los fines de fundar un nuevo saber de orden trascendente sino de poner de relieve, más bien, la necesidad tanto del pensamiento metafísico como de una práctica artística que transcienda los límites de la mera autorreferencialidad formal. Así entendida, la afirmación de Henrich con respecto al carácter limitado de la conciencia coincidiría con su referencia a la imposibilidad de superar de una manera definitiva la tensión que se establece entre las dos tendencias fundamentales de la subjetividad, esto es, entre la autoconsciencia y la autoconservación. Desde la perspectiva de este autor, si el pensamiento metafísico y la práctica artística continúan siendo imprescindibles, pese a los avances de las ciencias sociales y naturales, es porque no es posible evadir la necesidad de conducir la vida de una manera consciente. De hecho, para Henrich, conducir la vida de una manera consciente significa asumir la tarea de articular en una unidad vital y reflexiva las autodescripciones contradictorias a las que da lugar la tensión constitutiva de nuestra subjetividad. Por ello mismo, la condena que realiza Henrich de las perspectivas positivistas o naturalistas actuales -dentro de las que incluye planteos como los de Habermas (HENRICH, 1988, 24s)- tiene un doble carácter: ellas no solo anularían la tensión entre la autoconsciencia y la autoconservación, procurando reducir la primera a la segunda, sino que negarían el momento especulativo que se encontraría contenido en la propia decisión de hacer prevalecer este último principio por sobre la autorreferencialidad de la instancia subjetiva (HENRICH, 1982, 164s).

\section{El arte como principio de articulación de una vida consciente}

Dejando de lado la problemática del pensamiento metafísico, que ocupa un lugar central en la perspectiva de Henrich y que -como lo pone en evidencia su análisis de la obra de Hölderlin- no siempre es posible diferenciar con claridad de sus reflexiones estéticas, a continuación nos concentraremos en estas últimas. En este sentido, resulta necesario antes que nada reconstruir sus consideraciones generales acerca del arte, para poder analizar, en un segundo momento, la especificidad que el autor le atribuye al arte moderno en lo que respecta a la configuración de una vida consciente. De manera tal que, en términos generales habría que decir que Henrich le atribuye al arte un fundamento de carácter antropológico. La continuidad de la dimensión artística, que opera como supuesto de toda la reflexión de 
Henrich, se sostiene sobre el reconocimiento de la necesidad de que las concepciones opuestas de nosotros mismos, a la que da lugar nuestra naturaleza doble, sean integradas en el pensamiento y en la praxis vital (HENRICH, 2003, 294). Esto liga desde el comienzo la concepción de Henrich a ciertas posiciones que, sin renunciar a la diferenciación que esferas que ha traído aparejada la emergencia del arte moderno, ${ }^{21}$ le asignan a la dimensión artística una función de orden compensatorio. ${ }^{22}$

En este sentido no solo llama la atención el hecho de que Henrich le adjudique al arte en general la tarea de orientar el desarrollo de una vida consciente y de que, de manera consecuente, rastree en el arte autónomo la persistencia de elementos que serían propios de configuraciones artísticas de carácter preautónomo. ${ }^{23}$ Más allá de esto, lo que vincula a Henrich con las perspectivas compensatorias es su tendencia a enfatizar la especificidad que asume el arte moderno en el marco del proceso de integración. Para Henrich, el establecimiento de una subjetividad libre habría producido una serie de mejoras para la vida del hombre: la profundización de la comprensión psicológica e histórica, la humanización de los castigos y el desarrollo de un ordenamiento político que haría posible las diferencias individuales. No obstante, este proceso también habría dado lugar a un estado general de confusión y habría intensificado y modificado, por ello mismo, las exigencias de orientación que, desde siempre, pesaban sobre la dimensión artística. Las habría intensificado, porque la propia emergencia de una subjetividad libre habría desplazado a las instituciones (la iglesia, el estado o la propia filosofía) que hasta el momento habían realizado la tarea de integración, ${ }^{24}$ y las habría modificado, además, porque, en el nuevo

\footnotetext{
${ }^{21}$ Henrich reconoce en numerosas oportunidades el carácter irrenunciable de la autonomía del arte. Por ejemplo, cuando sostiene que las obras de arte solo puede tener una resonancia para la vida subjetiva en función "de su compleja construcción y del hecho de que se hallan más allá de las realización cotidianas y de las rutinas del arte utilitario". (HENRICH, 2003, 29).

22 Estrictamente la noción de compensación se aplica a los miembros del Collegium Philosophicum de Münster: Joachim Ritter, Odo Marquard y Hermann Lübbe. Aquí la utilizo en un sentido general para hacer referencia a aquellas perspectivas que consideran que el arte o la cultura pueden operar de contrapeso frente a los daños que causa el proceso de modernización.

23 "Que el arte intervenía en la vida en tanto totalidad y ella se encontraba en relación con una manera de pensar y una praxis, que iba más allá de las cosas cotidianas, es un hecho evidente tanto para las culturas como para el pensamiento temprano." (HENRICH, 2003, 270)

24 "En una época en la cual los sujetos han sido liberados de tales normas [las que imponían las antiguas instituciones], ellos se encontrarán arrojados a un camino que los conduce por experiencias en las cuales ellos son caen en conflictos de autointerpretación con independencia de qué alternativa elijan" (HENRICH, 2003, 26). En este contexto Henrich hace referencia a la frase de Albert Camus según la cual si el mundo fuera claro, no existiría el arte. Aparentemente, por medio de esta cita, Henrich procura poner en evidente el carácter crítico de su concepción estética. No obstante, Henrich no tiene en cuenta ni el estatuto que posee la oscuridad del mundo en su propia concepción filosófica ni la relación
} 
contexto histórico, la integración ya no podría ser pensada a costa de las diferencias individuales y del dinamismo de la vida subjetiva. En este sentido sostiene Henrich: "solo así los sujetos acceden a una forma de interpretación que ya no los coloca en una situación de mera aparente estabilidad, por medio de la represión forzada y atemorizante de alternativas." (HENRICH, 2003, 27)

Como aparentemente ya habría sido advertido por Hegel, esto último condena de antemano todos aquellos proyectos que se hallan orientados a restituir formas artísticas de carácter monumental. En este punto, Henrich se pliega a la tendencia generalizada de la estética del siglo XX a cuestionar la idea wagneriana de una obra de arte total (HENRICH, 2003, 39-64). Para Henrich, ante la propia emergencia de la subjetividad libre, la pretensión de recrear una obra de arte total solo podría asumir un carácter historicista o irónico, como en el caso de la arquitectura de Gaudi (HENRICH, 2003, 272). No obstante, el rechazo de las formas artísticas totalizadoras, no lo lleva a renunciar a toda posible configuración artística, de la misma forma que la imposibilidad de volver presente la subjetividad de una manera acabada no lo conduce a renegar ni de esta última ni de la posibilidad de establecer una relación entre ella y la producción artística en general.

Ciertamente, el arte moderno parte del presupuesto de que todo acceso a un ordenamiento de carácter absoluto debe fundarse en el movimiento vital de la conciencia. Esto torna inadecuadas las formas no problemáticas del arte clásico, pues el sujeto libre, lejos de disponer de una interpretación que le permita definir de antemano su lugar del mundo, debe producirla de manera subjetiva. Por ello mismo, la experimentación y el desarrollo de lineamientos novedosos juegan un rol determinante en el marco del arte moderno. Sin embargo, desde la perspectiva de Henrich, la preeminencia de estos elementos no debe lugar ni a obras de carácter absolutamente fragmentario ni a la disipación de las connotaciones existenciales de las búsquedas artístico-formales. No debe suponer la disolución de la configuración artística, porque el sujeto libre no renuncia a alcanzar la certeza de sí mismo en una configuración de carácter global, sino que aspira a lograrla por medio del movimiento conflictivo de su propia vida consciente. En términos artísticos esto se traduciría en la exigencia de

que él mismo establece entre esa oscuridad y la representación artística. Así, mientras Henrich concibe ontológicamente el carácter inaccesible del mundo y le asigna al arte la tarea de articular las tensiones que se desprenderían de este hecho, las posturas críticas se caracterizan, por lo general, por enfatizar la necesidad de pensar el mundo en términos históricos. Esto es particularmente evidente en el caso de un autor como Adorno, quien, por otra parte, no le adjudica al arte la función de tornar posible una vida con sentido, sino más bien la de manifestar de manera radicalizada el carácter problemático de las formas sociales existentes. En este sentido, Henrich podría ser incluido en la larga lista de autores alemanes que, desde Carl Schmitt en adelante, asumieron que, lejos de orientarse en términos utópicos, la tarea de la filosofía consistía en desarrollar estrategias que permitieran demorar la inminente llegada del apocalipsis. 
articular el libre descubrimiento de las posibilidades de los materiales y de los nuevos efectos estéticos con el imperativo de un equilibrio de carácter formal. Por ello mismo, sostiene Henrich, la autonomización de la dimensión estética no debe ser equiparada con la proclamación de la absoluta autorreferencialidad de los lenguajes artísticos. De hecho, para Henrich, el proceso de articulación del momento experimental con la máxima clasicista del equilibrio reflejaría el movimiento de integración que resulta constitutivo de la propia vida consciente (HENRICH, 2003, 30).

Para Henrich, tanto la resonancia subjetiva de la representación artística como la pretensión de totalidad continúan estando presente incluso en aquellas producciones autorreferenciales del arte modernista que parecen hallarse explícitamente orientadas a destruir toda expectativa de cierre formal. En este punto Henrich se encarga de aclararnos que dichas obras poseen una forma de funcionamiento levemente diferente a las del arte moderno clásico. Como lo pondrían en evidencian las sinfonías de Beethoven (HENRICH, 2003, 41) o la novela de formación de Goethe, en la modernidad clásica el movimiento subjetivo asumía la función de clarificar aquello que aparecía de una manera concreta e inmediata. La experimentación modernista, en cambio, buscaría superar o a trascender por medio de la representación artística el contenido de la experiencia empírica. En este sentido afirma Henrich:

\footnotetext{
Si en la época en la que la subjetividad fue puesta en libertad, el valor para la profundidad y la claridad en cuanto a la presentación de su desarrollo formal era un principio a partir del cual se creaba gran arte, el arte se encuentra hoy bajo el imperativo de la reserva y del compromiso reservado con la multiplicidad de distancias. (HENRICH, 2003, 37s)
}

De manera tal que, el arte modernista continuaría asumiendo la tarea de compensar la pérdida de experiencia subjetiva que habría traído aparejado el desarrollo del mundo moderno, pero introduciría una modificación importante en cuanto al modo de realizar dicha tarea. Esto es, al igual que ya sucedía en el arte moderno, en el caso del arte modernista los quiebres no serían introducidos si no a los fines de garantizar un mayor poder de integración. No obstante, el artista modernista -Henrich está pensando aquí en autores como Beckett o como Kafka- habría descubierto que aquello que debería ser representado en el plano artístico, esto es, el sí mismo del hombre, se encuentra más allá de toda posible objetivación. Por ello mismo, su trabajo no consistiría en colocar lo real en la esencialidad de su aparecer, sino en extraerlo de su forma habitual de aparición:

En su historia, el arte pudo ser entendido como una transfiguración de lo visible que desenvolvía el juego de las formas en el pathos de la reconciliación. El arte moderno introduce la "bella apariencia" en el límite en el cual se 
propaga la oscuridad y la monotonía. A estas últimas les gana terreno el arte moderno, lo cual remite al hecho de que el sí mismo es posible en función de fundamentos incomprensibles. (HENRICH, 2003, 140)

Desde la perspectiva de Henrich, el arte modernista reaccionaría así contra el peligro de una estetización de la vida cotidiana que se habría hallado contenido en la tendencia del arte clásico a presentarse como un modelo de la unidad vital. No obstante, Henrich no está dispuesto a interpretar el carácter negativo del arte modernista en términos de una renuncia a sus funciones de orden representativo, como hacen autores como Adorno. Para él, la deformación extrema de la representación tendría el objetivo de "dejar aparecer detrás de las superficies de la vida y del lenguaje algo inaprehensible e informulable," (HENRICH, 2008) es decir, el sî mismo reflexivo. "En la medida en que ella [la representación artística] vuelve visible el rasgo fundamental que gobierna todos los movimientos de la subjetividad, ella adquiere el carácter de un retroceso por detrás de la presencia cotidiana" (HENRICH, 2003, 141). De manera tal que, pese a su apariencia negativa, el arte modernista tendría un carácter doblemente afirmativo: él afirmaría tanto las funciones integradoras del arte como el poder de unificación que subyace el movimiento de la vida subjetiva, y lo haría en un contexto en el cual resulta necesario reconocer, por una parte, la imposibilidad de una presentación artística adecuada de la totalidad de la vida del hombre y, por la otra, la inexistencia de garantías metafísicas que permitan dar cuenta de las diversas autodescripciones subjetivas.

\section{Conclusión}

Resulta sintomático de la concepción estética de Henrich el hecho de que, pese a hacer referencia al riesgo de una estetización de la vida que se seguiría del modelo artístico clásico, finalmente asuma la decisión de defender la estética hegeliana, en vez de decidirse por aquella perspectiva que, según sus propias palabras, habría "enfrentado al idealismo estético de la vida quiebre formales calculados", (HENRICH, 2008), esto es, el romanticismo temprano alemán. Como ya anticipamos, los motivos que llevan a Henrich a admitir los quiebres solo a los fines de garantizar la profundización del poder de unificación, remiten más allá del orden estético. De hecho, solo en la medida en que la experimentación artística se ajuste a las necesidades de integración formal, la obra de arte puede hallarse en condiciones de satisfacer las dos exigencias que le impone Henrich a la dimensión estética. Esto es, puede recordarle al individuo que, en tanto sujeto, se halla obligado a conducir su vida en términos conscientes, y puede reforzar, además, la propia capacidad de integración que presupone la realización de esta tarea. 
El carácter apriorístico de la concepción estética que defiende Henrich se ve reflejado de una manera particularmente clara en su enfatización de la continuidad histórica del arte. Así, lejos de asumir el momento de ruptura que supuso el arte modernista, en tanto puesta en crisis de las formas representativas clásicas, Henrich descubre en él una radicalización del mismo presupuesto filosófico que habría operado de base del arte moderno, esto es, tanto el arte moderno como la producción artística del modernismo remitirían "a una mediación del sí mismo con el ser que no tiene lugar ni por medio de un movimiento autónomo del sí mismo ni por medio de una realidad inextricable que no resulta visible en términos fenoménicos." (HENRICH, 2003, 143) De esta forma, la reducción del arte modernistas a materiales simples tales como "la palabra, el sonido y el color" o en su tendencia a un orden no figurativo (HENRICH, 2003, 145-6), se hallaría orientada a poner de relieve el carácter no disponible del fundamento de la vida consciente. De esta forma, en un autor como Beckett, "que decía que uno debía interrumpir la arquitectónica de los lenguajes para permitir que emergiera aquello que estaba tras ellos" (HENRICH, 2003, 56), se evidenciaría, según Henrich, el carácter indisponible del fundamento de la subjetividad.

Si bien la pretensión de Henrich de incorporar el arte modernista en un relato de carácter histórico-compensatorio ya ejerce alguna violencia sobre la autonomía formal que sería propia de aquel, las dificultades de su planteo se tornan particularmente visibles cuando esta lectura se proyecta sobre el arte contemporáneo. En términos generales Henrich procura redescribir las formas artísticas contemporáneas de tal manera que estas puedan ser interpretadas en términos representativos, esto es, como representaciones del fundamento inescrutable de la subjetividad (HENRICH, 2008). No obstante, donde esta redescripción deja de ser posible, el autor asume de antemano una posición desnostativa. Esto sucede de una manera especialmente llamativa en su análisis de las tendencias desdiferenciadoras del arte contemporáneo. En efecto, ante la radicalidad de este fenómeno, Henrich se ve obligado a dejar de lado todo intento de redescripción y se pregunta, más bien, si la ausencia de la concentración no debería impedir la existencia de todo arte significativo. La conclusión a la que arriba nos revela hasta qué punto sus presupuestos filosóficos se presentan como un obstáculo a la hora de entender el arte contemporáneo. Desconociendo la especificidad de este último, Henrich convierte a la atención concentrada en un elemento indispensable para la apreciación del arte meritorio. Sin esta no se produciría, según Henrich, el aislamiento de la temática con respecto al entorno que hace posible el grado de intensidad que caracteriza al arte en sentido estricto. Concentrándose en la arquitectura, el autor sostiene al respecto: 
El efecto específico de la arquitectura tiene lugar al menos en igual medida por medio de experiencia que se hacen en movimiento por medio del espacio que por medio de la contemplación de cuerpos arquitectónicos... En lo que a mí respecta, considero por el momento que la concentración en la disposición y el posible énfasis en la experiencia se corresponden de manera necesaria. Así, la música significativa solo se pude receptar como tal en una concentración semejante. Si uno interrumpe esta última solo resta la producción sonora de un estado de ánimo, en el cual irrumpe todo tipo de asociaciones. (HENRICH, 2003, 290)

Henrich no advierte en este punto que la atención dispersa, que sería propia de las formas desdiferenciadas del contemporáneo, podría inducir una reorganización no convencional de la experiencia. ${ }^{25}$ Sin embargo, su omisión no posee un carácter meramente circunstancial. Ella pone de manifiesto, más bien, tanto el origen tradicional de su concepción artística como su tendencia a someter a la dimensión estética a argumentos de orden moral. De hecho, si la tendencia del arte contemporáneo a poner en suspenso el concepto de totalidad resulta cuestionable para Henrich no es en virtud de un análisis propiamente estético, sino más bien de la incapacidad de las formas artísticas desdiferenciadas para reflejar la dinámica total de la subjetividad (HENRICH, 2001, 226) Desde la perspectiva del autor, el arte contemporáneo no se halla en condiciones de compensar la crisis de identidad subjetiva a la que habría dado lugar el proceso moderno de racionalización. En virtud de su propio carácter disperso, la producción artística contemporánea establecería un vínculo con la subjetividad que se caracterizaría más bien por rebasar o desdibujar los límites de esta última que por apuntalar los procesos subjetivos de integración.

Como lo ponen en evidencia las propias dificultades que enfrenta la estética contemporánea a la hora de interpretar este fenómeno en términos unívocamente progresistas, ${ }^{26}$ Henrich parece hallarse en lo cierto cuando

\footnotetext{
${ }^{25}$ Utilizamos el término "atención dispersa" en el sentido crítico en que lo usa Benjamin en su famoso ensayo "La obra de arte en la época de su reproductibilidad técnica". Esto es, dispersión no sería equivalente en este punto a mera diversión. Lo que estaría en cuestión sería el tipo de experiencia que hacen posible aquellos objetos que contradicen las jerarquías significativas establecidas hasta el punto de volver indecidibles sus propios límites con respecto al ámbito no propiamente artístico. Dejamos de lado la acepción crítica que el propio Benjamin le atribuye a dicha noción en sus trabajos acerca de Brecht (EILAND, 2010, 53-74).

${ }^{26}$ Pienso sobre todo en las nuevas generaciones de la teoría crítica, esto es, en autores como Christoph Menke (MENKE, 2013) o Juliane Rebentisch (Rebentisch, 2012). Mucho más
} 
desconfía de la posibilidad de establecer a priori el sentido ético-político de la conmoción de las subjetividades establecidas que tendría lugar en el ámbito estético. No obstante, la referencia al riesgo que conllevaría la experiencia estética liberada de las determinaciones ético-políticas no parece ser suficiente para legitimar la tentativa de Henrich por eliminar la ambigüedad que habría sido constitutiva del arte moderno. De hecho, todo parece indicar que el potencial estético de este último encontraba su fundamento en su propia capacidad para poner en suspenso las formas sociales establecidas. En este sentido, podríamos decir que, en su intento por recuperar un concepto alternativo de la "subjetividad", Henrich acaba reconstruyendo una versión cercenada del arte y del sujeto moderno. Se trataría de una visión que, si bien remueve sus escollos, acaba renunciando también a su antiguo componente crítico; a un componente crítico del que probablemente daban cuenta de una manera más fidedigna las tensiones de la estética hegeliana entre el conocimiento de la apariencia estética y su concepción idealista de la misma. En realidad, sería esta tensión, y no un mero capricho personal, lo que reflejaría la particular estima que manifestaba Hegel frente a dos fenómenos artísticos tan contradictorios entre sí como podían serlo la pintura holandesa, que convertía a la apariencia estética en su propio objeto de determinación, y el humor objetivo, que apuntaba a mostrar el triunfo de la idea incluso en medio de lo ocasional. La interpretación de Henrich, en cambio, se halla orientada a neutralizar esta ambiguiedad y a someter al arte moderno al imperativo unilateral de la integración.

consecuente en este punto es el teórico de la literatura Karl Heinz Bohrer (BOHRER, 1981). 


\section{Bibliografía}

BAUEMLER, A. Das Irrationalitätsproblem in der Ästhetik und Logik des 18. Jahrhunderts bis zur Kritik der Urteilskraft. Darmstadt: WBG, 1975.

BOHRER, Karl Heinz, Plötzlichkeit. Zum Augenblick des ästhetischen Scheins. Frankfurt: Suhrkamp, 1981.

CASSIRER, E. La filosofía de la Ilustración. Trad. E. Imaz. México: FCE, 1981.

CRAMER, K. 'Erlebnis': Thesen zu Hegels Theorie des Selbstbewußtseins mit Rücksicht auf die Aporien eines Grundbegriffs nachhegelscher Philosophie. En H. G. Gadamer (ed.). Stuttgarter Hegel-Tage 1970, Beiheft 11, Bonn: Hegel-Studien, 1974, p. 537-603.

DEWS, Peter. The Limits of Disenchantment. Londres y Nueva York: Verso, 1995.

EILAND, Howard. Recepción en la dispersión. En: USLENGHI, Alejandra (ed.). Walter Benjamin: Cultura de la imagen. Traduccción de Alejandra Uslenghi y Silvia Villegas. Buenos Aires: Eterna Cadencia Editora, 2010, p. 53-74.

FICHTE, J. G. Versuch einer neuen Darstellung der Wissenschaftslehre: Vorerinnerung, Erste und Zweite Einleitung, Erstes Kapitel (1797/98). Hamburg: Meiner, 2014.

FRANK, M. Einführung in die frühromantische Ästhetik. Frankfurt: Suhrkamp. 1989.

FRANK, M. La piedra de toque de la individualidad. Reflexiones sobre sujeto, persona e individuo con motivo de su certificado de defunción postmoderno. Trad. Claudio Gancho. Barcelona: Herder, 1995.

FRANK, M. Selbstbewusstseinstheorien von Fichte bis Sartre. Frankfurt: Suhrkamp, 1993.

FRANK, M. Was ist Neostrukturalismus? Suhrkamp: Frankfurt, 1983.

FRANK, M. Welche Gründe gibt es, Selbstbewusstsein für irreflexiv zu halten. En ProtoSociology - Essays on Philosophy. 2013, p. 1- 21. Disponible en http://www.protosociology.de/on-philosophy.htm

GETHMANN-SIEFERT, Annemarie. Drama oder Komödie? Hegels Konzeption des Komischen und des Humors als Paradigma der romantischen Kunstform. En A. Gehtmann-Siefert, B. Collenberg-Plotnikov \& L. De Vos (Eds.), Die geschichtliche Bedeutung der Kunst und die Bestimmung der Künste. Hegels Berliner Ästhetikvorlesungen im Kontext der Diskussion um die Grundlagen der philosophischen Ästhetik, München: Wilhelm Fink, 2005a, pp. 175-188

GETHMANN-SIEFERT, Annemarie. Hegel über Kunst und Alltäglichkeit. En A. Gethmann-Siefert y Ursula Franke (ed.), Kulturpolitik und Kunstgeschichte: Persepektiven der Hegelschen Ästhetik. Hamburgo: Meiner, 2005b, pp. 37-64.

HABERMAS, J. Nachmetaphysisches Denken. Frankfurt: Suhrkamp, 1988. 
HABERMAS, J. Rückkehr zur Metaphysik: Eine Tendez in der deutschen Philosophie? En Merkur 39, 1985, p. 898-905.

HABERMAS, J.; HENRICH, D. Zwei Reden. Aus Anlass des HegelPreises. Frankfurt: Suhrkamp, 1974.

HEGEL, G. W. F. Lecciones sobre la estética. Traducción Alfredo Brotóns Muñoz, Madrid: Akal, 1989.

Hegel, G. W. F. Solgers nachgelassene Schriften und Briefwechsel. En:

Werke. Band 11, Frankfurt: Suhrkamp, 1979, p. 205-235.

HEIDEGGER, M. Nietzsche, Pfullingen: Neske, 1961.

HENRICH, D. Denken und Selbstsein, Vorlesungen zur Subjektivität. Frankfurt: Suhrkamp, 2007.

HENRICH, D. Die Grundstruktur der modernen Philosophie. En: H. Ebeling (ed.), Subjektivität und Selbsterhaltung: Beiträge zur Diagnose der Moderne, Frankfurt: Suhrkamp, 1976, p. 97 - 144.

HENRICH, D. Dieter Henrich im Gespräch mit Renate Solbach. Zukunft im Enden. En Labis, 2008. https://themen.iablis.de/2008/henrich08.html

HENRICH, D. Fichtes ursprüngliche Einsicht. En D. Henrich y H. Wagner (eds.) Subjektivität und Metaphysik. Klostermann: Frankfurt, 1966, p. 188 232.

HENRICH, D. Fixpunkte. Abhandlungen und Essays zur Theorie der Kunst, Frankfurt: Suhrkamp, 2003.

HENRICH, D. Fluchtlinien, Frankfurt: Suhrkamp, 1982.

HENRICH, D. Hegel en su contexto. Traducción Jorge A. Díaz A., Venezuela: Monte Ávila, 1990.

HENRICH, D. Konzepte. Essays zur Philosophie in der Zeit. Frankfurt: Suhrkamp, 1988.

HENRICH, D. „Selbstbewusstsein. Kritische Einleitung in eine Theorie“. En R. Bubner, K. Cramer, R. Wiehl (eds.), Hermeneutik und Dialektik. Aufsätze 1: Methode und Wissenschaft. Lebenswelt und Geschichte. Tübingen: Mohr, 1970, p. 257-284.

HENRICH, D. „Selbstsein und Bewusstsein“, en e-Journal Philosophie der Psychologie, 8, 2007, p. 1-19. http://www.jp.philo.at/texte/HenrichD1.pdf.

HENRICH, D. Über die Einheit der Subjektivität. En Philosophische Rundschau Vol. 3, No. 1/2, 1955, pp. 28-69.

HENRICH, D. Versuch über Kunst und Leben, München/Wien: Hanser, 2001.

HÖLDERLIN, F. Juicio y ser. En Ensayos, trad. Felipe Martínez Marzoa, Madrid: Hiperión, 1983, p. 25-35.

MENKE, Christoph, Die Kraft der Kunst, Frankfurt: Suhrkamp, 2013

REBENTISCH, Juliane, Die Kunst der Freiheit, Frankfurt: Suhrkamp, 2012.

ŽIŽEK, Slavoj Goza tu síntoma, Jacques Lacan dentro y fuera de Hollywood. Traducción por Horacio Pons. Nueva Visión: Buenos Aires, 1994.

Autor(a) para correspondência: María Verónica Galfione, Bv. Pellegrini 2750, S3000 Santa Fe, Argentina. veronicagalfione@yahoo.com.ar 